\title{
474185 - AVOIDANCE OF PREOPERATIVE FASTING BY HYPOCALORIC NUTRITION STIMULATES ALBUMIN SYNTHESIS AND REDUCES MUSCLE CATABOLISM AFTER SURGERY
}

\author{
Ralph Lattermann, MD, $\mathbf{P h D}^{1}$, Sarkis Meterissian, MD $\mathrm{MSc}^{2}$, Olasunkamni \\ Adegoke, $\mathrm{PhD}^{3}$, Errol Marliss, $\mathrm{MD}^{4}$, Hanan el-Shobary, $\mathrm{MD}^{1}$, Franco Carli, $\mathrm{MD}$ \\ MPhil $^{1}$, Linda Wykes, PhD $^{5}$, Thomas Schricker, MD PhD ${ }^{1}$ \\ 1. Department of Anaesthesia, McGill University Health Centre, Montreal, QC, \\ Canada \\ 2. Department of Surgery, McGill University Health Centre, Montreal, QC, Canada \\ 3. School of Kinesiology \& Health Science, York University, Toronto, ON, Canada \\ 4. Nutrition and Food Science Center, McGill University, Montreal, QC, Canada \\ 5. School of Dietetics and Human Nutrition, McGill University, Montreal, QC, \\ Canada
}

Introduction: Perioperative administration of hypocaloric nutrition, initiated 20 hours before surgery, attenuates whole body protein catabolism after colorectal surgery as assessed by stable isotope infusions of L-[1-13C]leucine. Whole body protein kinetics do not provide insight into organ specific metabolic pathways. The goal of the present study was to investigate the effect of preoperative hypocaloric nutrition on protein metabolism at the organ level, i.e. hepatic synthesis rates of albumin and fibrinogen and muscle protein synthesis two days after colorectal surgery. Cellular events in muscle were analyzed by measuring mRNA expression of ubiquitin and two of its key ligases.

Methods: After approval from the local Ethics Committee and after obtaining written informed consent, sixteen patients undergoing colorectal cancer surgery were randomly assigned to receive intravenous hypocaloric nutrition starting either 20 hours before the operation or with surgical skin incision. Nutrition was administered until the second postoperative day, with glucose providing $50 \%$ and amino acids $20 \%$ of each patient`s measured resting energy expenditure. Fractional and absolute synthesis rates of albumin, fibrinogen and muscle protein were determined using primed continuous infusions of L[2H5]phenylalanine two days after the operation. The expression of mRNA of proteolytic genes in muscle (MAFbx/atrogin-1, ubiquitin, MuRF-1) was determined by quantitative reverse transcriptase polymerase chain reaction. Statistical analysis was performed by Student's t-test and Mann-Whitney U test where appropriate. Correlations were calculated with either Pearson's or Spearman's Correlation Coefficients, as appropriate.

Results: Fractional ( $38 \pm 11$ vs. $18 \pm 4 \% / \mathrm{d}, \mathrm{p}<0.05)$ and absolute albumin synthesis rates $(292 \pm 81$ vs. $126 \pm 31 \mathrm{mg} / \mathrm{kg} / \mathrm{d}, \mathrm{p}<0.05)$ were higher in the preoperative feeding group. No difference was observed in the fractional synthesis rates of fibrinogen (99 \pm 32 vs. $93 \pm 20$ $\% / \mathrm{d}, \mathrm{p}>0.05)$ and muscle protein (1.9 \pm 0.4 vs. $2.2 \pm 0.8 \% / \mathrm{d}, \mathrm{p}>0.05)$. The expression of MAFbx/Atrogen-1 (95 \pm 78 vs. 46 \pm 32 , $\mathrm{p}>0.05$ ) and ubiquitin (881 \pm 262 vs. $662 \pm 312$, $\mathrm{p}>0.05$ ) tended to be higher in fasting patients, while the expression of MuRF-1 was significantly elevated (5.65 \pm 4.63 vs. $2.18 \pm 0.97, \mathrm{p}<0.025)$. Significant positive correlations were observed among all three: MAFbx vs ubiquitin ( $r=0.732, \mathrm{p}=0.003$ ), and vs MuRF-1 ( $r=0.763, \mathrm{p}=0.002)$ and MuRF-1 vs ubiquitin ( $r=0.591, \mathrm{p}=0.026)$, across all 
16 muscle samples.

Discussion: Avoidance of preoperative fasting by hypocaloric nutrition initiated 20 hours before colorectal surgery stimulates postoperative albumin synthesis and decreases muscle catabolism as reflected by reduced proteolytic gene expression. Fibrinogen and muscle protein synthesis are not affected.

\section{References: .}

